\title{
Belice, textos de su historia
}

Mónica Toussaint Ribot

L a bibliografía para el estudio de la historia de Belice es poco extensa, sobre todo si la comparamos con la de otros países de América Latina y el Caribe como Brasil, Argentina, Venezuela, Perú, Guatemala, Cuba o Puerto Rico, para los cuales encontramos una amplia variedad de fuentes que abordan los temas más diversos, tanto políticos como económicos y sociales.

En el caso de Belice no sólo nos enfrentamos a la escasez de las fuentes, sino a lo restringido de la temática de las mismas. Gran parte de los textos están dedicados al estudio del conflicto diplomático sobre la soberanía de Belice, en el cual están implicados Inglaterra, España, México y Guatemala. En ellos se reproducen y analizan una serie de tratados y convenciones que intentan establecer de manera definitiva a quién pertenece el territorio beliceño. Además, encontramos una serie de trabajos de autores mexicanos, guatemaltecos y británicos cuyo interés fundamental es la defensa de los derechos de cada uno de sus países de origen sobre Belice.

De manera casi constante, la visión histórica de Belice ha sido una visión "desde fuera". Quizás una de las características que definen a la historiografía de Belice ha sido la ausencia de historias "nacionales". Es más, los primeros intentos en este sentido se convirtieron en historias prohibidas que presentaban una versión contraria a los intereses británicos.

Como consecuencia de lo anterior podemos situar grandes vacios históricos en cuanto a temas y épocas. Destacan, sobre todo, la ausencia de trabajos de historia social y la escasez de textos de historia económica, y abundan, como ya dijimos, los estudios que abordan la historia política, especialmente desde el punto de vista diplomático.

A partir del proceso de independencia ha surgido un interés renovado por un país con el cual tanto mexicanos como centroamericanos estamos profundamente relacionados, pero del que conocemos muy poco. De aquí que se hayan elaborado una serie de estudios referentes al sindicalismo, los partidos políticos y el proceso independentista, junto con diversos artículos de corte antropológico que analizan problemas específicos, sobre todo en el ámbito de la cultura. No obstante, el afán de acercarse a la realidad beliceña se ha sustentado en la importancia geopolítica del territorio y no tanto en la intención de dar a conocer la historia del pueblo beliceño; más bien, ha sido éste un pueblo olvidado por los historiadores, los cuales poco se han preocupado por su estudio y la difusión de su historia.

La presente bibliografía ccmentada se agrupa en seis temas:

1) Obras generales. Se trata de narraciones de hechos históricos, geografía, economía, administración pública, costumbres, etc. Fundamentalmente, son obras descriptivas, aunque algunas de ellas tocan aspectos explicativos o casuales. Entre las más completas destacan las de Stephen Caiger, Narda Dobson y María Emilia Paz.

2) Informes oficiales. A pesar de ser escuetos y algunos de ellos 
cargados de tecnicismos, ofrecen al lector una buena información, sobre todo en lo que se refiere a datos estadísticos. Con todo, no dejan de ser la versión oficial de la historia de Belice.

3) Relatos de viajeros. Constituyen valiosos testimonios de la época, los cuales presentan diversos puntos de vista que corresponden a la nacionalidad del autor, a los objetivos de la visita y a los intereses específicos tanto personales como políticos. Merecen especial mención las amenas obras de Squier y Stephens así como el relato de sir Daniel Morris, este último de gran importancia, no sólo para la historia sino también para la botánica.

4) Obras y documentos dedicados al estudio de la relación entre Belice y México. Es el más numeroso conjunto de obras, el cual a su vez puede subdividirse en tres grupos. El primero, cuyo eje es la defensa de los derechos de México sobre Belice; aquí destacan las obras de Carlos Echánove, Isidro Fabela y Gustavo Pérez Trejo. El segundo, que aborda el problema de la relación Belice-México a raíz de la guerra de castas; es particularmente importante el informe que el gobernador Joaquín Baranda envía al gobierno federal. Y el tercero, formado por una serie de documentos, tratados y correspondencia diplomática referentes a la cuestión limítrofe.

5) Obras que hacen referencia al conflicto anglo-guatemalteco por la soberanía de Belice. Además de los textos dedicados al estudio de la pugna anglo-guatemalteca, encontramos también literatura apologética de la soberanía guatemalteca sobre el territorio beliceño. Entre las principales obras destacan las de Roberto Bardini, Francis Gall, Pedro González Blanco y Marco Augusto Recinos.

6) Artículos de corte antropológico. Abordan,temas particulares tales como la lingüística, las festividades autóctonas, la historia de la región fronteriza entre Belice y Quintana Roo y el problema de la cultura beliceña; tal vez los más especializados son los textos del doctor Grant D. Jones.

Finalmente, resulta pertinente aclarar que para la elaboración de este comentario bibliográfico sólo se consultaron las principales bibliotecas de la ciudad de México y no se hace referencia a textos o documentos de los que conocemos su existencia en otros acervos - como la Biblioteca del Congreso en Washington o los Archivos del Foreign Office de Londres- pero que no llegaron a nuestras manos. Tampoco se consultó de manera directa el Archivo Genaro Estrada de la Secretaría de Relaciones Exteriores; sin embargo, gran parte de los documentos ahí resguardados se encuentran ya publicados en varias de las colecciones documentales que aquí se presentan.

Sirva éste como un primer acercamiento para todos aquellos interesados en reconstruir la historia beliceña.

\section{Obras generales}

$\mathrm{BIM}$

972.82

CAl.b

COLMEX

972.82

C133b
Caiger, Stephen L., British Honduras, Past and Present, Londres, Allen \& Unwin, 1951.

Basado en los Archivos de Honduras Británica, 16701884 de sir John Burdon, el autor hace un detallado recuento de la historia de Belice. Tomando como punto de partida la llegada de Peter Wallace al territorio, Caiger relata el origen del asentamiento de cortadores de made- 
ra, los conflictos con España, la transformación del asentamiento en colonia británica, así como la controversia con Guatemala. También se hace referencia a algunos aspectos de la historia beliceña durante la primera mitad del presente siglo. Incluye una cronología, un mapa de Honduras Británica y uno de Centroamérica.

BIM

972.8203

CAL.b

BNAL

972.82

CAL.b

COLMEX

972.82

$\mathrm{C} 146 \mathrm{~b}$
Calderón Quijano, José Antonio, Belice, 1663 (?)-1821: historia de los establecimientos británicos del río Valis, hasta la independencia de Hispanoamérica, Sevilla, Escuela de Estudios Hispanoamericanos de la Universidad de Sevilla, 1944, 503 p.

Extenso estudio que aborda la historia de Belice a partir de tres aspectos fundamentales: el histórico, el jurídico y el internacional. La finalidad del libro es dar a conocer Belice a través de la documentación de archivos españoles; logra reconstruir la historia beliceña de manera detallada, a lo largo de tres siglos. Incluye varios apéndices con documentos y mapas. Resulta curioso que la periodización utilizada por el autor se base en las administraciones de los reyes españoles.

\begin{abstract}
BFCPUNAM Clegern, Wayne M., British Honduras: Colonial Dead F1446 $3 \mathrm{c} 4$

COLMEX 972.82 C624b

FLACSO

321.03

C624b End, 1859-1900, Baton Rouge, Louisiana State University Press, 1967, $214 \mathrm{p}$.

El autor rescata la historia de Belice de la segunda mitad del siglo XIX, escasamente tratada en otros textos. Fundamentalmente hace referencia a los siguientes aspectos: la transformación del asentamiento británico de cortadores de palo de tinte en la colonia de Honduras Británica, los conflictos de límites con México, el auge y declive de la producción forestal, la crisis económica y la búsqueda de nuevos caminos para su desarrollo económico. Este trabajo está basado en la tesis doctoral de Clegern, The International Role of British Honduras, 1859-1900, Berkeley, University of California, 1959. Cuenta con un apéndice de oficiales británicos y de funcionarios en Honduras Británica.
\end{abstract}

BIM

972.82

DOB.h

FLACSO

320.97282

D635h

Dobson, Narda, A History of Belize, Londres, Longman Caribbean, 1973, $361 \mathrm{p}$.

Uno de los estudios más completos sobre la historia de Belice, este trabajo se remonta a los orígenes del asentamiento de cortadores de madera, pasando por su constitución en colonia, hasta los años previos a la independencia. Tres siglos de historia beliceña se recogen en sus aspectos político, económico y social, con base en documentos y textos consultados en bibliotecas y archivos ingleses, jamaiquinos y beliceños. Contiene varios mapas y se incluyen diversos apéndices, entre ellos, un listado de gobernantes, un cuadro estadístico poblacional y un breve comentario bibliográfico. duras, Montreal, Provincial Publishing Co., 1946, 116 p. 
COLMEX

972.82

D687h

Estudio general que aborda la historia beliceña a través de dos grandes ejes: primero, un panora ma global del desarrollo interno de Belice a lo largo de casi cuatro siglos, que incluye los aspectos políticos, económicos, culturales, religiosos, educativos y geográficos; segundo, un seguimiento de la controversia entre Guatemala e Inglaterra, desde el periodo colonial hasta mediados del siglo $\mathrm{xx}$.

BIM Gibbs, Archibald R., British Honduras: a Historical and 972.82 Descriptive account of the Colony from its Settlement, GIB.b 1670, Londres, Sampsonhow, Marston, Searle and Rivington, $1883,198 \mathrm{p}$.

BNAL

972.92

Síntesis histórica y económica de Belice que aporta GIB.b una buena cantidad de datos. Hace referencia a la guerra de castas en Yucatán, a las relaciones entre los colonos beliceños y los indios de Chan Santa Cruz y al comercio de armas.

BFLUNAM

F1449

B7H84

COLMEX

972.8203

H927d

IPGH

$327(72.82)$

H

$\mathrm{BIM}$

972.82

PAZ.b

COLMEX

972.82

P3487b

Humphreys, Robert A., The Diplomatic History of British Honduras, 1638-1901. Londres, Oxford University Press, $1961,196 \mathrm{p}$.

Presenta la historia de Belice como asentamiento de los cortadores de palo de tinte al tiempo que relata los conflictos entre España e Inglaterra en torno a la soberanía sobre el territorio beliceño. Igualmente se habla de las reclamaciones guatemaltecas sobre esta región y se incluye gran cantidad de información sobre los problemas fronterizos con Yucatán y los tratados de límites.

Paz Salinas, María Emilia, Belice, el despertar de una nación, México, Siglo XXI, 1983, 192 p.

Con el fin de apoyar y justificar la soberanía y el derecho a la autodeterminación del pueblo beliceño, la autora aborda diversos temas de la historia y del presente beliceños: Belice y su integración al marco del capitalismo dependiente, la génesis de la nacionalidad beliceña, su configuración estructural, el proyecto de desarrollo, Belice en la encrucijada internacional, su lucha por la Independencia en el marco de la descolonización y el caso de Belice en los foros internacionales. Destaca la utilización de la fuente hemerográfica así como de la documentación de Naciones Unidas, Secretaría de Relaciones Exteriores y Foreign Office de Londres.

BIM

972.8205

PRA.V

COLMEX 972.82 P112v
Prats y Beltrán, Alardo, Visión actual de Belice, México, Libro Mex., 1958, 194 p.

Recuento de la historia de Belice en el siglo xx, su desarrolo, su lucha por la independencia y los problemas contemporáneos con México y Guatemala. Incluye una breve reseña histórica de la creación de Belice y un apéndice sobre el tratado de límites con México. Se anexan fotografías. 
BLC

Quilés. Inés María, "Belice: de los bucaneros ingleses a los marinos norteamericanos", Economía Informa, México, Facultad de Economia de la LNam, septiembre de 1984, p. 27-36.

En este articulo la autora presenta un esbozo general del proceso histórico de Belice a partir de su independencia con el fin de dar a conocer un pais tan cercano a México y del cual se sabe tan poco. Así, el objetivo del texto es analizar los aspectos que puedan ser de interés para México a fin de mejorar con ello las relaciones económicas, políticas y sociales entre ambos países. El trabajo se divide en los siguientes apartados: breve recuento histórico de Belice, panorama general actual, importancia de Bèlice para México, importancia estratégica militar de Belice para Estados Unidos.

BIM

972.82

VIV.b

Vivó, Jorge A., Belice: país y pueblo; evolución económica y política, México, unam, sobretiro del Anuario de Geografía, $1967,88 \mathrm{p}$.

Breve texto que aporta abundante información sobre Belice en torno a los siguientes aspectos: rasgos geológicos y geomorfológicos, antecedentes históricos, relaciones de Belice con México y Guatemala, composición étnica y aspectos político-sociales, evolución económica y evolución política. En el texto abundan los mapas, cuadros estadísticos y gráficas. Se incluyen referencias bibliográficas por temas.

BIM Waddell, D.A.G., British Honduras, a Historical and Con972.82 temporary Survey, Londres, Oxford University Press, WAD.b $1961,151 \mathrm{p}$.

Extenso estudio que pretende ofrecer al lector un acercamiento a la realidad beliceña, aportando gran cantidad de datos. Hace referencia a cuatro aspectos fundamentales: 1) la historia social, económica, diplomática y constitucional; 2) la tierra y la gente; 3) la economía; 4) las relaciones políticas y la politica exterior. Incluye mapas.

\section{Informes oficiales}

$\begin{array}{ll}\text { BIM } & \text { Burdon, sir John, Brief Sketch of British Honduras, Past, } \\ \text { 330.97282 } & \text { Present and Future, Londres, The West India Committee, } \\ \text { BUR.b } & 1928,53 \mathrm{p} .\end{array}$

COLMEX Informe elaborado en 1926 por el gobernador de la 972.82 colonia con el fin de difundir su historia y dar a conocer B951.a sus recursos y posibilidades futuras. Contiene información sobre la geografía, la historia, la administración, la industria, el comercio y las finanzas. Se anexa un mapa de Belice.

BIM

972.82

MIS.1

Colonial Office, British Honduras, Report for the Year 1955, Londres, Her Majesty's Stationery Office, 1957, $94 \mathrm{p}$.

Informe general que aporta gran cantidad de datos y cifras sobre los siguientes temas: población, empleo, finanzas, comer- 
cio, producción agrícola, forestal, minera, pesquera y manufacturera; educación, vivienda, salud, legislación, prensa y administración. Incluye un mapa del territorio, cuadros estadísticos y fotografías.

BIM

972.82

MIS. 1

Colonial Office, British Honduras, Report for the Years 1964 and 1965, Londres, Her Majesty's Stationery Office, $1968,115 \mathrm{p}$.

Informe general que ofrece gran cantidad de datos y cifras sobre los siguientes aspectos: población, empleo, finanzas, comercio, producción agrícola, forestal, minera, pesquera y manufacturera; educación, vivienda, salud, legislación, prensa y administración. Incluye cuadros estadísticos, fotografías y un mapa del territorio.

COLMEX Gregg, Algar Robert, British Honduras, Londres, Her 972.82 $\mathrm{G} 819 \mathrm{~b}$ Majesty's Stationery Office, 1968, 158 p.

Descripción general del territorio y la población, que forma parte de una serie de informes al gobierno británico acerca de sus colonias, su forma de vida y la manera como se gobiernan. Hace referencia a la historia temprana, el desarrollo político y el problema con Guatemala. Además, se presenta un panorama de la ciudad de Belice y otros pueblos, los diferentes grupos étnicos, los principales productos, el comercio y las finanzas. Incluye un buen mapa de Belice.

\section{Relatos de viajeros}

BIM

917.28

HAE.V

Haefkens, Jacobo, Viaje a Guatemala y Centroamérica, Guatemala, Editorial Universitaria, 1969, 342 p.

Jacobo Haefkens, cónsul general de Holanda, visitó

Centroamérica de 1826 a 1829 . Esta obra constituye el resultado de sus observaciones sobre la política centroamericana, incluyendo abundantes referencias a las ciudades y lugares, estilo de vida, diversiones y tipos humanos. Contiene además un informe sobre la colonia inglesa de Belice. La presente edición reproduce los grabados originales del autor sobre la ciudad de Guatemala, personajes típicos populares y un mapa de Centroamérica en 1832.

COLMEX Morris, sir Daniel, The Colony of British Honduras, its 972.82 Resources and Prospects; with Particular Reference to its M875c Indigenous Plants and Economic Productions, Londres, Edward Stanford, 1883, $152 \mathrm{p}$.

Enviado por el gobierno británico a Honduras Británica, Morris hace una vívida descripción de la flora beliceña así como de sus principales productos, como la caoba, la caña de azúcar y el cacao. Según el propio autor, el objetivo de la obra consiste simplemente en realizar un recuento de los recursos existentes en la colonia con el fin de auxiliar a todos aquellos interesados en su desarrollo. Contiene un apéndice bibliográfico botánico dedicado a los plantadores. 
BIM

917.28

SQU.s
Squier, Ephraim George, The States of Central America, Nueva York, Harper \& Brothers, Publishers, 1858, 781 p.

Diplomático norteamericano, Squier viajó a Centroamérica a mediados del siglo XIX con el claro objetivo político de propiciar un acercamiento con el gobierno de Nicaragua - con base en el gran interés de los Estados Unidos en la construcción de un canal interoceánico-y tratar de reducir la influencia británica en la región. En su libro, Squier relata sus impresiones sobre la geografía, el clima, la población, los recursos económicos, el comercio, la organización política y la religión. Se incluye un pequeño capítulo sobre Belice. Destacan los mapas, los dibujos y las reproducciones de litografías que ilustran la obra.

BIM Stephens, J. L., Incidentes de viaje en Centroamérica,

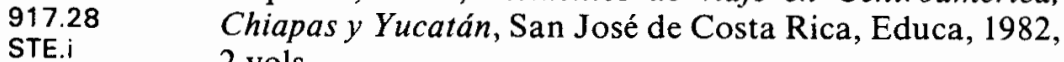
2 vols.

El autor, de origen norteamericano, recorre el istmo centroamericano en 1839 para cumplir una misión diplomática encomendada por el presidente Van Buren. La obra contiene importantes testimonios sobre aspectos diversos: las ruinas mayas, la guerra federal, los caudillos, el paisaje, los centros urbanos, las costumbres. En el primer capítulo se describe la llegada de Stephens a Belice. Además, la obra se ilustra con los dibujos del arquitecto Frederick Catherwood.

BIM Swan, Michael, British Honduras, Londres, Phoenix Hou972.82 MIS.1 se, $1957,39 \mathrm{p}$.

Este pequeño relato del viajero Michael Swan ofrece un panorama general de algunos elementos básicos que permiten al lector acercarse a la historia de Belice, la geografía, la economía, la gente, el recuento de los acontecimientos políticos y económicos entre 1900 y 1957 y la administración de la colonia. Presenta también un capítulo sobre el futuro de Belice para la segunda mitad del siglo $\mathrm{xx}$.

BIM

917.281

WIN.g

Winter, Nevin Otto, Guatemala and her People of To-day, Boston, The Page Company Publishers, 1909, 307 p.

El autor, de origen norteamericano, pretendió ofrecer un relato veraz - basado en su estancia en Centroamérica a principios de este siglo y ayudado con lecturas complementarias sobre la región- con el fin de cubrir la necesidad de la gente de Estados Unidos, cada vez más interesada en "los otros americanos" que hablan lenguas latinas. Resultado de esta inquietud, su obra presenta una fiel descripción de la tierra, los recursos naturales, la historia, la gente, las costumbres, las ciudades. Un capítulo está dedicado a Honduras Británica. Cuenta además con una buena cantidad de ilustraciones. 


\section{Obras y documentos dedicados al estudio de la relación entre Belice y México}

BNAL Aspiroz, Manuel, "Establecimiento inglés en Belice", BoB letín de la Sociedad Mexicana de Geografía y Estadística, 910.6272 México, 2a época, vol. 4, septiembre, 1872, p. 698-715.

Traducción del francés con fecha 20 de agosto de 1787, en el presidio de San Felipe de Bacalar de Yucatán. Incluye tres documentos: acta de entrega del territorio de Belice a los ingleses firmada por Edouard Despard y Henri de Grimarest, teniente del rey de Campeche; extracto del expediente relativo a la colonia de Belice o Wallis y apuntes históricos sobre Honduras Británica.

$\begin{array}{lll}\text { BIM } & \text { FLACSO } & \text { Asturias, Francisco, Belice, s.p.i., } 92 \text { p. } \\ 972.82 & 972.8203 & \\ \text { AST.b } & \text { A859b } & \end{array}$

BNAL IPGH Discurso del doctor Francisco Asturias $972.82 \quad 972.82 \quad$ leído en la sesión pública del 5 de abril de AST.b $\quad$ A 1925 , día de su ingreso a la Sociedad de Geografia e Historia de Guatemala. El texto se refiere a tres puntos, básicamente: el origen de Belice, la situación de Centroamérica a mediados del siglo XIX y el origen de las pretensiones de México sobre el Petén y Belice. Se anexa un pequeño apartado de descripción geográfica.

BNAL

082.1

MIS.103

Baranda, Joaquín, La cuestión de Belice, Campeche, Sociedad Tipográfica, 1873, $126 \mathrm{p}$.

Informe al gobierno federal del gobernador de Campeche, Joaquín Baranda, en el cual relata la situación de Belice durante la guerra de castas, las actividades de los mayas rebeldes de Chan Santa Cruz y su relación con los colonos beliceños. En el apéndice se incluyen importantes documentos sobre conflictos de límites y tráfico de armas, entre otros.

BFLUNAM Castellanos, Francisco Xavier, La intendencia de Yucatán F1376

C38 y Belice, México, s.e., 1962, 62 p.

COLMEX 972.82 $0349 i$ territorios adyacentes.
Contiene información sobre la intendencia de Yucatán y Belice, el obispado de Yucatán, el territorio que hoy ocupa Belice y el origen de esta colonia. Se incluye también un análisis jurídico del conflicto fronterizo con Belice y Guatemala. Se anexa un mapa de Yucatán y los $106 \mathrm{p}$. 


$\begin{array}{ll}\text { BNAL } & \text { IPGH } \\ 972.8202 & 972.8 \\ \text { COS.b } & \text { C }\end{array}$

Trabajo presentado por el autor en el vili Congreso Mexicano de Historia celebrado en 1947 , en el que se relatan el descubrimiento, la exploración, la conquista, la colonización y la evangelización con el fin de presentar una justificación histórica de la soberanía de España sobre el territorio de Belice. Contiene once anexos documentales.

BIM

972.82

$\mathrm{ECHt}$

Echánove Trujillo, Carlos A., Una tierra en disputa (Belice

BNAL

972.83

ECH.t ante la historia), Mérida, Editorial Yucatanense-Club del Libro, 1951, 171 p.

El autor, originario de Yucatán, emprende la defensa de los derechos de México sobre Belice con el argumento de que Gran Bretaña robó Belice a España, a México y a Guatemala. De aquí que en el texto se concluya la necesaria restitución de la parte del territorio beliceño correspondiente a México, no sólo como una forma de reparación material sino también moral. La obra hace referencia a las expediciones y ataques piratas en las costas de Yucatán y Campeche, a la creación del asentamiento de cortadores, a los diversos intentos de expulsar a los piratas del área, así como a los diferentes tratados celebrados entre España e Inglaterra a lo largo del siglo xviII. Incluye un apéndice documental.

$\begin{array}{ll}\text { BFCPUNAM } & \text { COLMEX } \\ \text { F1440 } & 972.82 \\ \text { B7F3 } & \text { F1 14b } \\ & \\ \text { BIM } & \text { IPGH } \\ 972.82 & 341.2(72.82) \\ \text { FAB.b } & \text { F }\end{array}$

Fabela, Isidro, Belice: Defensa de los derechos de México, México, Mundo Libre, 1944, $423 \mathrm{p}$.

Una de las obras más completas sobre el problema de Belice. Contiene datos y documentos relativos a los derechos de España sobre Belice; Belice como parte

BNAL

972.82

FAB.b de la Capitanía General de Yucatán; los derechos de México; las relaciones MéxicoInglaterra y el conflicto México-Guatemala. Se anexan también documentos sobre la definición de límites. La obra tiene el objetivo de justificar la reclamación de México sobre la parte del territorio de Belice que pertenecía a la Capitanía de Yucatán, y el reclamo de Guatemala sobre la porción correspondiente a la Capitanía de Guatemala. Además, se incluye un mapa de los ríos Valiz, Nuevo y Hondo, así como fotografias e ilustraciones.

BIM

972.82

HUB.b

BNAL

972.64

HUB.b

IPGH

972.82

$\mathrm{H}$
Hübbe, Joaquin, Belice, Mérida, Compañía Tipográfica Yucateca, 1940, $164 \mathrm{p}$.

El objetivo del autor, alemán naturalizado yucateco, consiste en dar a conocer la geografia, la economía, la historia y la organización política de Belice con el fin de que Yucatán pudiera defenderse de los posibles afanes expansionistas británicos. La obra que originalmente apareció en forma de artículos en el diario yucateco $\mathrm{El}$ Eco del Comercio, entre 1880 y 1881 , contiene diversos datos sobre los cacicazgos mayas independientes. Una 
buena parte de la información contenida en el texto fue tomada del informe del secretario colonial de Belice, Henry Fowler, de 1879.

BNAL

972.08842

Lafragua, José María, e Ignacio Vallarta, Correspondencia diplomática cambiada entre el gobierno de la República $y$ el de su Majestad Británica con relación al territorio llamado Belice, 1872-1878, México, Ignacio Cumplido, 1878, 94 p.

Colección de documentos que conforman la correspondencia y que aportan una buena información sobre los acuerdos diplomáticos a los que deseaban llegar México y Gran Bretaña. En la obra destaca la carta dirigida por Ignacio Vallarta a Lord Derby en 1878, en la cual se hace una defensa de los derechos de México sobre Belice a la vez que se acusa a Inglaterra de prolongar la guerra de castas al solapar a los colonos beliceños que venden armas a los mayas rebeldes.

BNAL

327.72097282

Mariscal, Ignacio, Informe del C. Ignacio Mariscal, MEX.i secretario del Despacho de Relaciones Exteriores, rendido ante el Senado, acerca del Tratado de Límites entre Yucatán y Belice, México, F. Díaz de León, $1893,55 \mathrm{p}$.

El propósito de este informe es dar una justificación a la firma del tratado de límites entre México y Belice en 1893. Para ello, se hace un recuento histórico desde la fundación de Belice en el siglo xvir, con el fin de demostrar que dicho territorio no pertenecía a la Capitanía de Yucatán. Además, se argumenta la necesidad de suprimir el tráfico de armas entre los colonos beliceños y los mayas rebeldes, por medio de un control riguroso de la línea fronteriza.

BIM Martínez Alomia, Santiago, Belice, Campeche, Biblio327.728107282 teca de El Reproductor Campechano, 1945, 374 p.

MAR.b

BNAL

327.7282097281

Originalmente publicado en forma de artículos en MAR.b el semanario El Reproductor Campechano, es este un amplio estudio histórico, político y legal sobre el tratado de limites concertado entre el licenciado Ignacio Mariscal, secretario de Relaciones Exteriores de México, y sir Spencer St. John, ministro plenipotenciario de Inglaterra, por medio del cual el gobierno mexicano reconoció a Inglaterra soberanía territorial sobre Belice. Incluye un vasto apéndice documental.

COLMEX Martínez Palafox, Luis, La cuestión de Belice, México, 972.82 M3856c Editorial Polis, 1945, 135 p.

Relación documental sobre la historia de Belice, desde el punto de vista mexicano, que pretende acudir a las fuentes originales para estudiar la historia, básicamente en el archivo de la SRE y en el Archivo General de la Nación, ramo de historia. Consta de seis capítulos: geografía histórica, la Nueva España, las guerras de independencia, la vida independiente hasta 1876, el porfirismo y consideraciones. 
BNAL

972.82

PEN.h

IPGH

972

p

buen mapa de Belice.

$\begin{array}{ll}\text { BIM } & \text { COLMEX } \\ \text { 972.82 } & 972.64 \\ \text { PER.d } & \text { C3522 } \\ \text { BNAL } & \text { FLACSO } \\ \text { 972.82 } & 320.97282 \\ \text { PER.d } & \text { P438.d } \\ & \\ & \text { IPGH } \\ & 972.82 \\ & \text { P }\end{array}$

BIM

ROM.d

Romo García, Eloy, Los derechos de México sobre Belice, México, unam, 1942, 80. p.

Tesis de licenciatura cuyo objetivo es la defensa de los derechos de México sobre una parte del establecimiento de Belice. El texto se divide en dos partes: 1) la histórica, en la que se hace referencia al origen del establecimiento de Belice, a los tratados anglo-españoles, a la jurisdicción de la Capitania General de Yucatán, al tratado entre México e Inglaterra de 1826, al tratado de límites de 1893 y a las pretensiones de Guatemala; 2) la jurídica, que trata la situación jurídica del problema, la reivindicación de Belice por Guatemala y la administración provisional de Belice.

BNAL

$\mathrm{R}$

327.72097283

MEX.t

Tratado de límites entre los Estados Unidos Mexicanos y Honduras Británica, seguido de los principales textos que a él se refieren, México, SRE, 1897, 139 p.

Contiene el texto del Tratado Mariscal-Saint John firmado en 1893, tanto en inglés como en español, acompañados de un mapa de Honduras Británica elaborado en 1860. Se incluyen también otros documentos al respecto, entre ellos, el Dictamen de la Comisión de Relaciones del Senado, dos informes del secretario de Relaciones Exteriores y una Defensa del Tratado.

BIM

Yucatán y Belice. Colección de documentos importantes que se refieren al Tratado del 8 de julio de 1893 celebrado entre el Sr. Lic. Ignacio Mariscal, en representación de México y Sir. Spencer Saint John, en representación de la Gran Bretaña, Mérida, Tipografía de G. Canto, 1894,172 p.

Se incluyen documentos de la Legislatura del estado de Yucatán dirigidos al gobierno federal relativos al problema de Belice, así como de la junta auxiliar de Mérida de la Sociedad Mexicana de Geografía y 
Estadística. Aparecen también los textos en que los ayuntamientos y juntas municipales de Yucatán piden al Senado se ratifique el tratado de límites con Belice. Se anexan como respaldo las opiniones de la prensa yucateca, favorables al tratado. Contiene, además, un mapa del territorio ocupado por la colonia de Belice, elaborado en 1893.

\section{Obras que hacen referencia al conflicto anglo-guatemalteco por la soberanía de Belice}

COLMEX Bardini, Roberto, Belice, historia de una nación en movi972.82 B246b miento, Tegucigalpa, Editorial Universitaria, 1978, 207 p.

FLACSO

320.97282 B246b
El texto es resultado del viaje realizado por el autor en 1977, por encargo del Comité para la paz entre los pueblos de Centroamérica, para analizar la situación de Belice, investigar acerca del reclamo territorial de Guatemala y realizar entrevistas políticas. Hace referencia a los siguientes aspectos: las guerras y los acuerdos entre 1660 y 1821 ; la herencia de España, la doctrina Monroe, el tratado de límites de 1859 , la convención de 1863 y el nacimiento de la nueva nación. Destacan las entrevistas a los miembros de la oposición y al primer ministro George Price.

BIM

972.82

GUA.b

Belice es de Guatemala, Guatemala, Secretaría de Información de la Presidencia de la República, 1958, 127 p.

Colección de documentos reunidos por el gobierno guatemalteco con un doble objetivo: reiterar la soberanía de Guatemala sobre la totalidad del territorio de Belice, de acuerdo con sus derechos históricos y jurídicos, y rechazar las pretensiones de México y Gran Bretaña sobre Belice, desde su punto de vista totalmente infundadas.

COLMEX

972.8203

$\mathrm{B} 577 \mathrm{~b}$

Bianchi, William J., Belize, Nueva York, Las Americas Publishing Co., 1959, 142 p.

Relación de la controversia entre Guatemala y Gran Bretaña acerca del territorio de Honduras Británica, con base en los principales tratados diplomáticos: Godolphin, París, Versalles, Londres, Amiens, Madrid y la Convención de 1859. Se incluye un capítulo especial sobre el Libro Blanco de Guatemala.

BIM

972.82

MIS. 1

Breve resumen de la disputa guatemalteca con la Gran Bretaña sobre el territorio de Belice (1783-1975), Guatemala, Ministerio de Relaciones Exteriores, 1976, 26 p.

Como su nombre lo indica, este texto constituye una síntesis que permite ubicar de manera rápida tanto los principales tratados celebrados entre Inglaterra y Guatemala con respecto a Belice, como los momentos históricos cruciales y los puntos de conflicto. 
BNAL

972.82

GAL.b

El estudio elaborado por Francis Gall, basado en documentos auténticos y jurisprudencia continentalmente aceptada, constituye un esfuerzo más por demostrar que Belice es parte integrante del territorio guatemalteco, descartando a su vez la pertenencia a Inglaterra y a México. Para ello, el texto se divide en tres partes principales: la geográfica, la histórica y el origen de las pretensiones de México sobre Belice. Cuenta con un anexo cartográfico con 24 mapas. Asimismo, al final de la obra se reproducen los tratados anglo-españoles de 1783 y 1786, el tratado de límites con México de 1882, la Convención de 1859 y el decreto que declara caduca dicha convención de cesión territorial debido al incumplimiento de la cláusula VII por parte de los ingleses.

BIM

972.82

GON.p

COLMEX

972.82

G6431p

González Blanco, Pedro, El problema de Belice y sus alivios, México, s.e., 1950, 129 p.

Apología de la soberanía guatemalteca sobre el territorio beliceño. El argumento principal del texto radica en explicar que sólo por la fuerza Belice se encuentra en poder de Gran Bretaña. La obra se centra, consecuentemente, en la disputa internacional sobre Belice, haciendo referencia a los tratados anglo-españoles, a los tratados anglo-americanos, al tratado de 1859 y a los supuestos derechos de México sobre Belice. Presenta además una relación sumaria de lo que opinan sobre el caso de Belice diversos internacionalistas iberoamericanos.

$\begin{array}{ll}\text { BIM } & \text { IPGH } \\ 972.82 & 327 .(72.82) \\ \text { MEN.i } & \text { M }\end{array}$

COLMEX

327.7282

M539.i
Mendoza, José Luis, Inglaterra y sus pactos sobre Belice, Guatemala, Secretaría de Relaciones Exteriores, 1942, 287 p.

Estudio de los tratados entre Inglaterra y Guatemala relativos a Honduras Británica. El texto pretende defender el derecho de Guatemala a reivindicar íntegro el terri-

torio de Belice negando, consecuentemente, cualquier posible derecho de Inglaterra sobre el mismo.

BIM Recinos, Marco Augusto, Les droits de Guatemala sur 972.82 Belice, Paris, Publicaciones de la Delegación de GuateMiS.1 mala, 1948, $32 \mathrm{p}$.

Defensa de la soberanía guatemalteca sobre el territorio de Belice que hace referencia a los tratados anglo-españoles y a los tratados anglo-guatemaltecos, en particular al de 1859. Contiene un mapa de la república de Guatemala que muestra la localización del territorio de Belice de acuerdo con las concesiones de 1783 y 1786.

\section{BIM}

917.281 ROD. 1

Rodriguez Beteta, Virgilio, El libro de Guatemala Grande: Péten-Belice, Guatemala, s.e., 1951, 3 vols.

Para el autor el texto tiene un doble objetivo. En primer lugar, difundir entre los guatemaltecos el sentimiento de la imperiosa necesidad de dirigir la mirada a la mitad norte 
del país con el fin de explotar sus infinitos recursos naturales. En segundo lugar, demostrar que para desarrollar el norte guatemalteco resulta imprescindible recuperar el territorio de Belice. En este sentido se abordan los siguientes temas: el aprovechamiento que del suelo Péien-Itzá hicieron los mayas; el sistema de los ríos peteneros; el desarrollo de la agricultura y el comercio (interior y exterior) entre los antiguos peteneros, así como las condiciones peteneras en cuanto a su geología, su aspecto físico, paisaje geográfico, climas, flora y fauna. En el texto abundan los mapas y las ilustraciones.

BIM Rodríguez Beteta, Virgilio, La política inglesa en Cen327.114 troamérica durante el siglo XIX, Guatemala, Ministerio de ROD.p Educación Pública, 1963, 245 p.

A pesar de que en el título se hace referencia sólo al tema de la política británica hacia el istmo centroamericano, el eje del estudio es la pugna entre los Estados Unidos e Inglaterra por lograr la hegemonia política y económica en la región. Destacan como elementos centrales los siguientes: el proyecto del canal interoceánico, el tratado ClaytonBulwer, la invasión de William Walker y la lucha por Belice. Se incluye un apartado en el que se argumenta la nulidad de fondo del tratado de 1859.

BIM

$327420728 \quad$ América, Tegucigal Chatfield, consul britanico en Centro ROD.C

Rodríguez, Mario, Chatfield, cónsul británico en Centro América, Tegucigalpa, Banco Central de Honduras, 1970, $526 \mathrm{p}$.

Resultado de la inquietud del autor por analizar la influencia británica en América Central a lo largo del siglo XIX, esta obra constituye un estudio pormenorizado de la carrera pública de Frederick Chatfield, cónsul británico en Centroamérica. Destaca la utilización de fuentes primarias para la investigación, realizada ésta en América Central, Inglaterra, Bélgica y Francia.

\section{Artículos de corte antropológico}

BLC

Allsopp, SRR, "British Honduras, the Linguistic Dilemma", Caribbean Quarterly, Kingston, University of the West Indies, vol. II, núms. 3 y 4, septiembre-diciembre, 1965, p. 54-61.

A pesar de su corta extensión, el texto resulta de especial importancia por la temática que trata: el problema linguístico en Honduras Británica. Incluye un buen mapa sobre la distribución lingüística de la población.

BLC Jones, Grant D.,"Maya-Spanish Relations in Sixteenth Century Belize", Belcast Journal of Belizean Affairs, Belice, vol. 1, núm. 1, diciembre, 1984, p. 28-40.

Con un alto grado de especialización, el trabajo del doctor Jones se remonta a los origenes de la influencia española en el territorio beli- 
ceño, sumándose a la tesis que justifica la soberanía española sobre Belice, previa a la británica.

BLC

Jones, Grant D., “Mayas, Yucatecos and Englishmen in the Nineteenth Century, Fiesta System of Northern Belize”, Belizean Studies, Belice, BISRA, vol. 10, núms. 3-4, 1982, p. 25-42.

Detallada descripción de las festividades autóctonas en el norte de Belice en la cual se hace referencia a los diversos preparativos, los rituales religiosos y no religiosos, las danzas, corridas de toros y todo tipo de tradiciones culturales.

BLC

Macías Zapata, Gabriel Aarón, "Transformaciones histórico-regionales de la frontera México-Belice", La formación histórica de la frontera sur, México, CIESAs, 1985, p. 199-304.

En este texto se analizan los cambios históricos y políticos de la región fronteriza entre Belice y Quintana Roo. El autor desarrolla tres líneas principales: la primera, consiste en una descripción de la geografía y la cultura en la época prehispánica, durante la conquista y la colonización españolas; la segunda, se refiere a los conflictos entre España e Inglaterra frente a los cortadores de palo de tinte, principalmente durante la guerra de castas; finalmente, se trata el periodo del Porfiriato, la Revolución y los años posteriores a la Revolución, cuando se inicia el reparto de tierras y la reforma agraria en la región del río Hondo.

BLC

Sánchez, I. E., Belize and its Cultural Identity, Belmopan, mimeografiado, 1984, $23 \mathrm{p}$.

Se trata de dos ponencias reunidas en un solo trabajo. La primera se presentó en 1982 en el Congreso de Sociólogos Centroamericanos en San José de Costa Rica; la segunda fue expuesta en Chetumal, México, en la conferencia sobre problemas de las culturas de la frontera, la cual tuvo lugar en 1981. El problema cultural se aborda entonces en dos apartados: "Crisis of a People in Search of their National Identity" y "Problems of Culture and National Identity for the Frontier Mestizos".

\section{BIBLIOTECAS CONSULTADAS}

BfCPUnAM Biblioteca de la Facultad de Ciencias Políticas y Sociales de la Universidad Nacional Autónoma de México

BFLUNAM Biblioteca de la Facultad de Filosofía y Letras de la Universidad Nacional Autónoma de México

BIM

Biblioteca del Instituto de Investigaciones Dr. José María Luis Mora

BLC Biblioteca de Lorena Careaga

BNAL Biblioteca Nacional 
COLMEX Biblioteca Daniel Cosío Villegas de El Colegio de México

FLACSO Facultad Latinoamericana de Ciencias Sociales

IPGH Instituto Panamericano de Geografía e Historia 